\title{
Shenhua Tablet inhibits mesangial cell proliferation in rats with chronic anti-Thy-1 nephritis
}

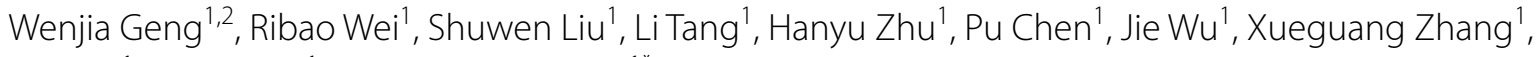
Fei Zhu' ${ }^{1}$ Zhong Yin ${ }^{1}$ and Xiangmei Chen ${ }^{*^{*}}$

\begin{abstract}
Background: In China, mesangial proliferative glomerulonephritis (MSPGN) is one of the most common kidney diseases. In this study, we treated a rat model of chronic anti-Thy-1 MsPGN with Shenhua Tablet and evaluated whether the tablet was able to protect the kidney function. Thirty-six Wistar rats were randomly divided into six groups: (1) Sham surgery (Sham); (2) anti-Thy-1 nephritis model (Thy-1); (3) anti-Thy-1 nephritis model + irbesartan-treated (Irb); (4) anti-Thy-1 nephritis model + low-dose of Shenhua Tablet (SHL); (5) anti-Thy-1 nephritis model + medium-dose of Shenhua Tablet (SHM); (6) anti-Thy-1 nephritis model + high-dose of Shenhua Tablet (SHH).

Results: Thirteen weeks after drug treatment, urinary proteins were quantified and renal pathological changes were thoroughly examined at the time point of $24 \mathrm{~h}$. Meanwhile, the expression levels of p-Erk1/2, cyclin D1 and p21 at the renal cortex were also tested. The levels of urinary proteins and total cholesterol in the blood were significantly reduced in rats treated with any drug tested in this study. The level of triglyceride was significantly reduced in all three Shenhua Tablet-treated groups. Renal pathomorphological scores were significantly improved in groups of Irb, SHM and SHH. Mesangial cell proliferation was significantly inhibited in any drug-treated group. p-Erk1/2 and cyclin D1 were downregulated whereas p21 was upregulated in the renal cortex.
\end{abstract}

Conclusions: Our study indicated that Shenhua Tablet is able to inhibit the abnormal proliferation of mesangial cells and to prevent kidney damage, which is likely associated with downregulation of $\mathrm{p}$-Erk $1 / 2$ and reduced activity of its downstream target-cyclin D1.

Keywords: Shenhua Tablet, Chronic anti-Thy-1 glomerulonephritis, Mesangial cell proliferation, Erk

\section{Background}

Mesangial proliferative glomerulonephritis (MsPGN) is characterized by widespread mesangial cell proliferation and an accumulation of extracellular matrix, and is the most common type of glomerulonephritis globally $[1,2]$. Abnormal proliferation of mesangial cells plays an important role in the onset and progression of glomerlular lesion [3]. Abnormally proliferative mesangial cells are

\footnotetext{
*Correspondence: xmchen301@126.com

1 Department of Nephrology, Chinese PLA General Hospital, Chinese PLA Institute of Nephrology, State Key Laboratory of Kidney Diseases, National Clinical Research Center for Kidney Diseases, Beijing Key Laboratory of Kidney Disease Research, Beijing 100853, People's Republic of China Full list of author information is available at the end of the article
}

able to release mediators of inflammation and mesangial matrix, which often cause severe glomerulosclerosis and interstitial fibrosis, resulting in irreversible progressive glomerulosclerosis, eventually leading to end-stage renal disease (ESRD) [4]. Therefore, to inhibit mesangial cell proliferation is a great therapeutic strategy for MsPGN and searching for such drugs is urgently needed for the treatment of this disease. Clinically, MsPGN patients especially IgAN patients, are currently treated with drugs such as hormones, cyclophosphamide, azathioprine, mycophenolate mofetil, leflunomide, and so on. However, these drugs often have toxic side effects, which largely compromise their clinical applications. Therefore, it has become critical to seek for effective treatments actively 
and further understand the mechanism underlying how these drugs protect the kidney functions [5, 6].

Shenhua Tablet has been widely used in clinical settings for many years. Clinical evidence has shown that this drug is able to reduce the level of proteinuria in patients with IgA nephropathy, and greatly improve the life quality of patients without serious adverse reactions [7]. However, whether Shenhua Tablet can protect the kidney functions of MsPGN patients remains completely unclear. This study aimed to investigate the role of Shenhua Tablet in the treatment of chronic anti-Thy-1 MsPGN, and to explore the possible mechanism underlying the improvement in kidney function after Shenhua Tablet treatment.

\section{Results}

\section{Shenhua Tablet reduces the proteinuria level in rats with chronic anti-Thy-1 nephritis}

The levels of urinary protein in $24 \mathrm{~h}$ were recorded for each group over the experimental process. We observed that the urinary proteins were gradually increased in each group after being given Thy- 1 antibody four times through intravenous injection at the time points W1, W3, W5 and W7 $(p<0.05)$. At the time point W11 (21 days after the last injection of Thy-1 antibody at W7), urinary proteins in $24 \mathrm{~h}$ in experimental groups were still significantly higher than that in the Sham group, indicating that the animal model of chronic anti-Thy-1 nephritis was reliably established $[8,9]$. At the last time point W20, the urinary proteins in $24 \mathrm{~h}$ in all drug-treated groups were significantly lower $(p<0.01)$ than that in the group of anti-Thy-1. However, there was no significant difference between SHM group and Irb group in terms of levels of urinary proteins in $24 \mathrm{~h}(p=0.14)$, which suggested that SHM and Irb functioned similarly in reducing the urinary proteins in $24 \mathrm{~h}$ for experimental rats (Fig. 1).

\section{The effect of Shenhua Tablet on the renal function and biochemical indices in blood}

The levels of serum creatinine in each group were also determined at the last time point W20. As observed, the level of serum creatinine in anti-Thy-1 group was significantly higher compared with that in the Sham group $(p=0.003)$. However, the levels of serum creatinine were not significantly changed $(p>0.05)$ in all drug-treated groups in contrast to that in the Thy-1 group, which demonstrated that irbesartan and Shenhua Tablet of any dose in this study were unable to reduce the level of serum creatinine in rats with anti-Thy-1 nephritis (Fig. 2).

At W20, the levels of serum albumin were not significantly increased $(p>0.05)$ whereas the levels of total cholesterol in blood were strikingly reduced $(p<0.05)$ in all drug-treated groups, compared with those in the Thy- 1 group. However, the levels of total cholesterol were not significantly different among all drug-treated groups $(p>0.05)$. It was noteworthy that Shenhua Tablet of any dose (low, medium or high) was able to reduce the levels of triglyceride significantly $(p<0.05)$. However, there was no significant differences when treated with different doses of the drug $(p>0.05)$ (Fig. 3).

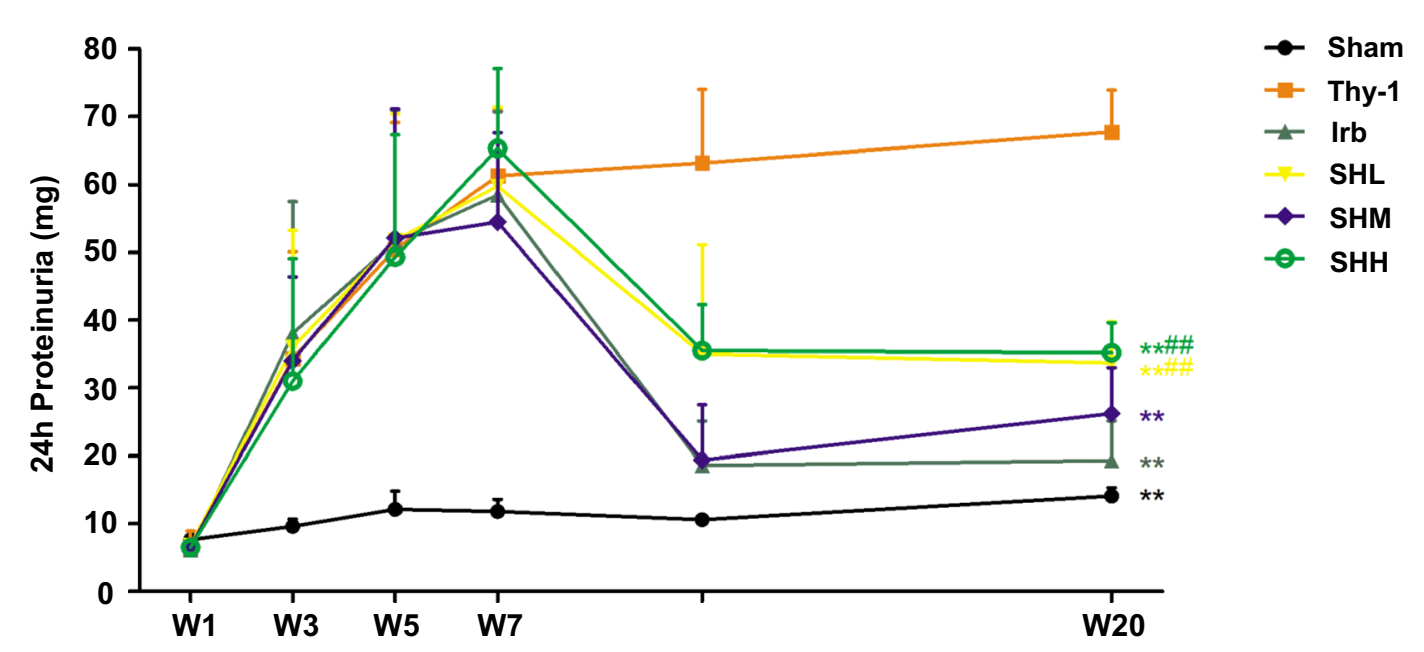

Fig. 1 The levels of urinary protein in $24 \mathrm{~h}$ were monitored for each group over the experimental process (week 1, 3, 5, 7, 11 and 20 after injection of Thy-1 antibody). At the last time point W20, the urinary proteins in $24 \mathrm{~h}$ in all drug-treated groups were significantly lower $(p<0.01)$ than that in the Thy-1 group. Values were expressed as mean $\pm \mathrm{SD} .{ }^{*} p<0.05,{ }^{* *} p<0.01 \mathrm{vs}$. Thy-1; ${ }^{\#} p<0.05,{ }^{\# \#} p<0.01 \mathrm{vs}$. Irb group 


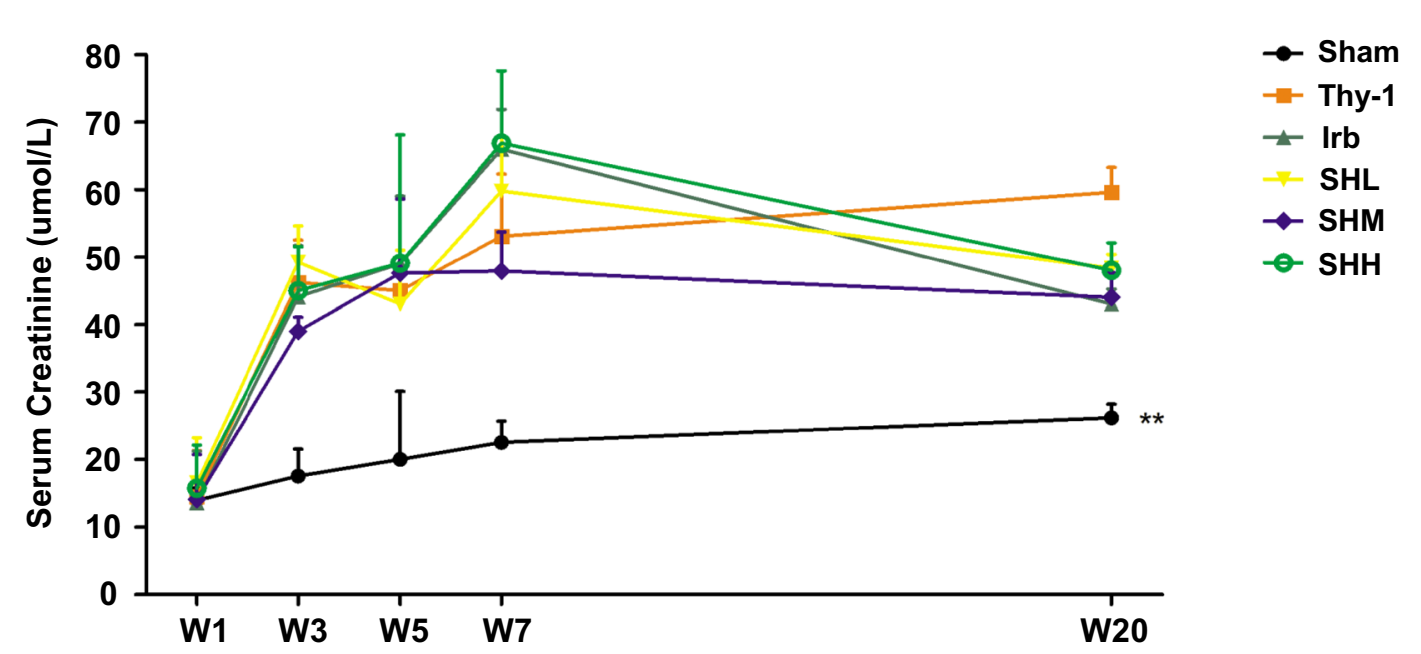

Fig. 2 The levels of serum creatinine in each groups at different time points. The levels of serum albumin were quantified in all six groups at different time points (week 1, 3, 5, 7 and 20 after injection of Thy- 1 antibody). Values were expressed as mean $\pm S D .{ }^{*} p<0.05,{ }^{* *} p<0.01$ vs. Thy-1

\section{Shenhua Tablet inhibits proliferation of mesangial cells in rats with anti-Thy- 1 nephritis and improves renal pathological scores}

The histopathological changes in glomeruli were examined under the microscope after PAS staining. At W20, the changes including increased proliferation of glomerular cells $(p<0.01)$ and mesangial cells, accumulation of mesangial matrix, widening of mesangial region, compression, deformation even occlusion of the capillary lumen, were observed in the Thy-1 group but not in the Sham group (Fig. 4a, b). Thirteen weeks after drug treatment, the proliferation of mesangial cells was significantly reduced; the widening of mesangial regions and accumulation of mesangial matrix were strikingly improved and the capillary lumen also recovered back to normal as compared with those in the Thy- 1 group (Fig. 4a). No increase in the number of mesangial cells and accumulation of mesangial matrix were observed in the Sham group (Fig. 4a). At W20, the number of glomerular cells in all drug-treated groups, including Irb $(p<0.05)$, SHL $(p<0.01)$, SHM $(p<0.01)$ and SHH $(p<0.01)$, was significantly reduced compared with that in the anti-Thy-1 group (Fig. 4a, b).

PCNA (proliferating cell nuclear antigen) is wellknown as a marker of cell proliferation which localizes in the nucleus. PCNA is barely detectable in the glomeruli of the Sham group. However, it was significantly increased at W20 in the Thy-1 group, compared with the Sham group $(p<0.01)$. Thirteen weeks after drug treatment, PCNA-positive cells were significantly reduced in all drug-treated groups when compared with those in the Thy-1 group $(p<0.01)$ (Fig. $4 \mathrm{c}, \mathrm{d})$.
Renal pathological score is generally used to comprehensively evaluate the kidney damage. At W20, the score was significantly higher in the Thy-1 group compared with that in the Sham group ( $p=0.001)$; However, this score was significantly decreased in three drug-treated groups, including $\operatorname{Irb}(p=0.01)$ SHM $(p=0.014)$ and SHH $(p=0.017)$, when compared with that in the antiThy-1 group (Fig. 4e).

\section{Shenhua Tablet downregulates $p$-Erk $1 / 2$ and cyclin D1 while upregulating p21 in the renal cortex of rats with anti-Thy-1 nephritis}

As Shenhua Tablet was able to inhibit the proliferation of mesangial cells, improve pathological scores and reduce the proteinuria level in rats with anti-Thy- 1 nephritis, we then went on to explore the possible molecular mechanism underlying how this drug worked. We first investigated the expression level of p-Erk1/2 to see whether Erk/Mapk signaling pathway was activated; we also examined cyclin D1 and p21 to see whether these cell cycle regulatory proteins were dysregulated. The data indicated that expression levels of p-Erk and cyclin D1 were strikingly increased in the Thy-1 group $(p<0.01)$ while the level of $\mathrm{p} 21$ was largely reduced $(p<0.05)$ when compared with the Sham group. However, in all groups with drug treatment, the expression levels of p-Erk were significantly reduced compared to that in the anti-Thy-1 group $(p<0.01)$. It is noteworthy that the expression levels of p-Erk in all Shenhua Tablettreated groups were even more reduced compared with that in the Irb-treated group. We also observed that in the drug-treated groups, the expression level of cyclin D1 was significantly decreased, compared with that in the Thy-1 


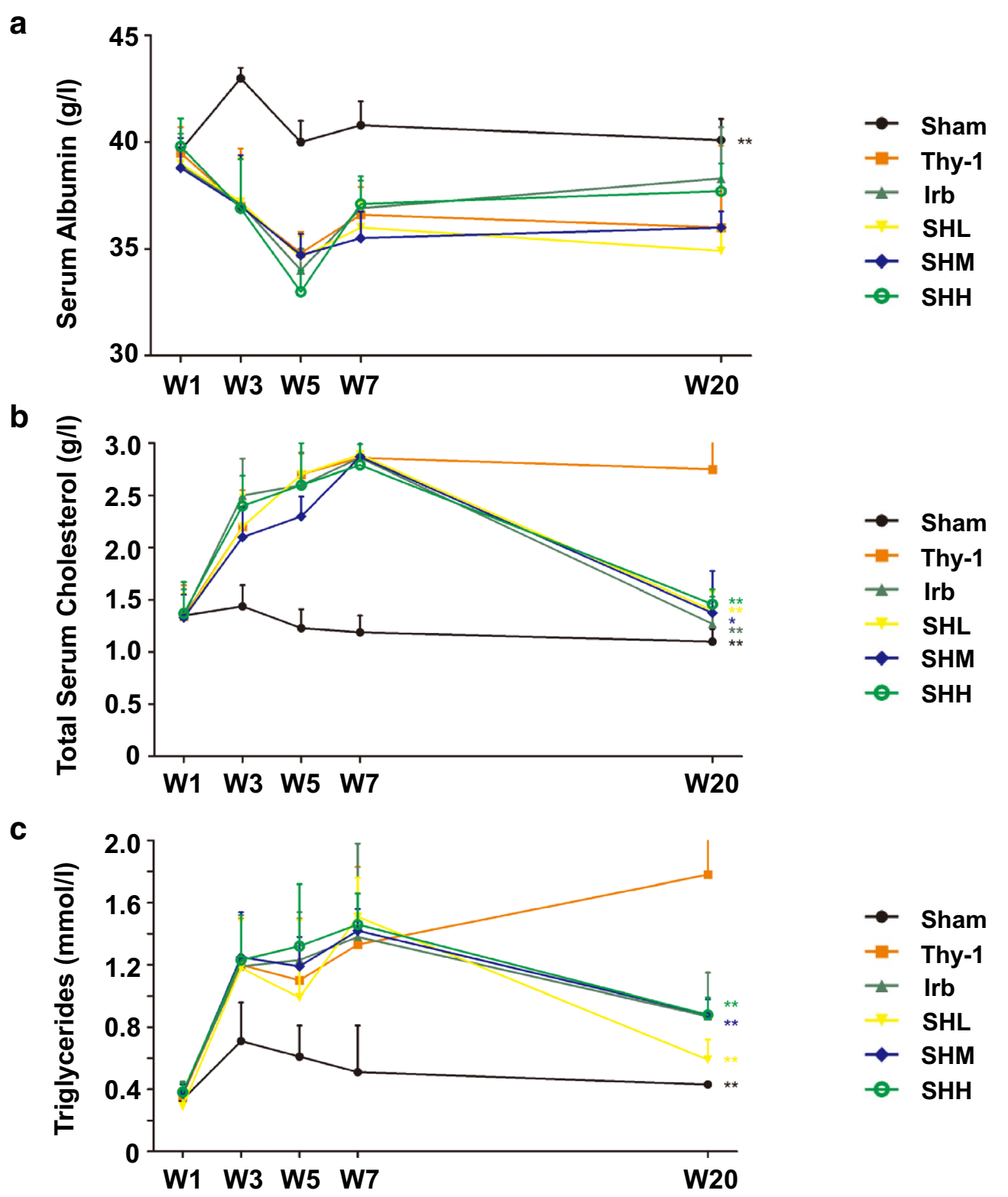

Fig. 3 The effect of Shenhua Tablet on the biochemical indices in blood. a The levels of serum albumin were quantified in all six groups at different time points (week 1, 3, 5, 7 and 20 after injection of Thy-1 antibody). b The levels of total cholesterol in blood were quantified in all six groups at different time points mentioned above. c The levels of triglyceride were quantified in all six groups at different time points mentioned above. Values were expressed as mean $\pm \mathrm{SD}$. ${ }^{*} p<0.05,{ }^{* *} p<0.01$ vs. Thy $-1 . \mathrm{n}=6$

group $(p<0.01)$. Moreover, we noticed that the expression of cyclin D1 was similar between the Irb group and SHM group, which suggested that Irb and Shenhua Tablet with medium dose had the same effect on the expression of cyclin D1. In addition, the expression levels of p21 in groups of Irb, SHL and SHM were significantly higher than that in Thy-1 group $(p<0.05)$ while there was no significant difference among all drug-treated groups $(p>0.05)$ (Fig. 5).

\section{Discussion}

Abnormal proliferation of mesangial cells is an important pathological feature of multiple immune-mediated glomerular diseases; continuous mesangial cell proliferation eventually leads to irreversible glomerulosclerosis, and even progresses to renal failure [4]. MsPGN, including IgAN and non-IgA MsPGN, is the most common glomerulonephritis and the primary cause of end-stage renal 
a

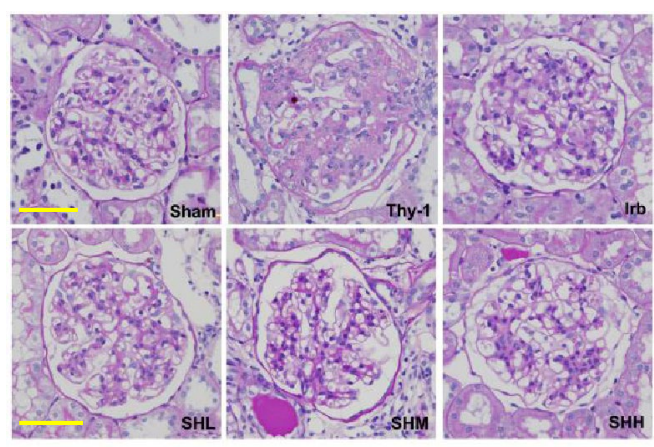

b

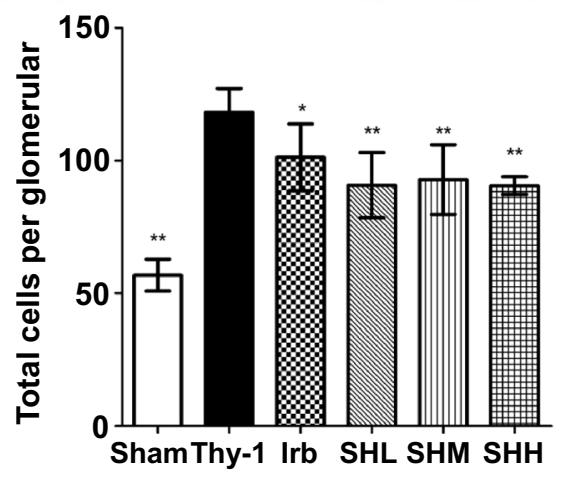

e

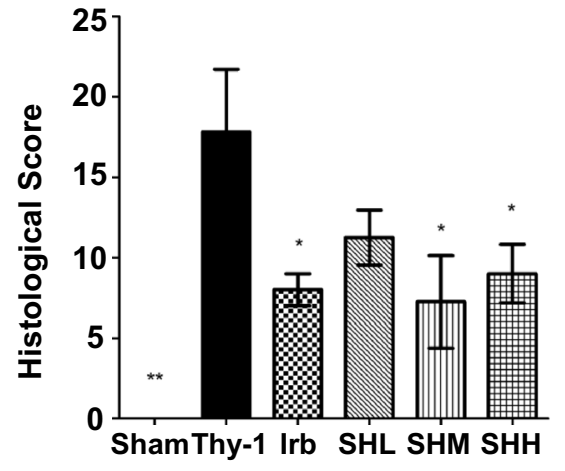

C

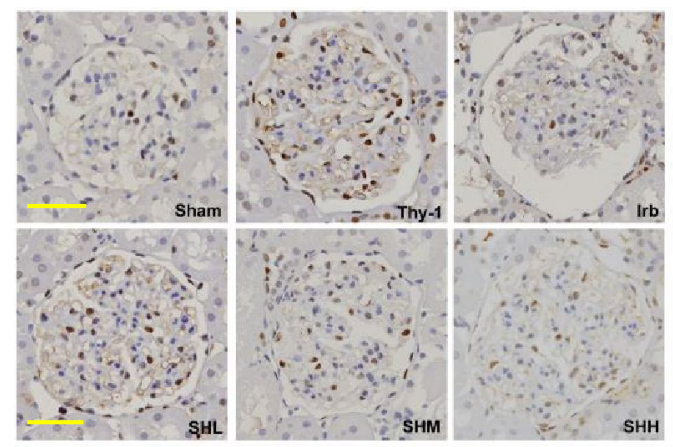

d

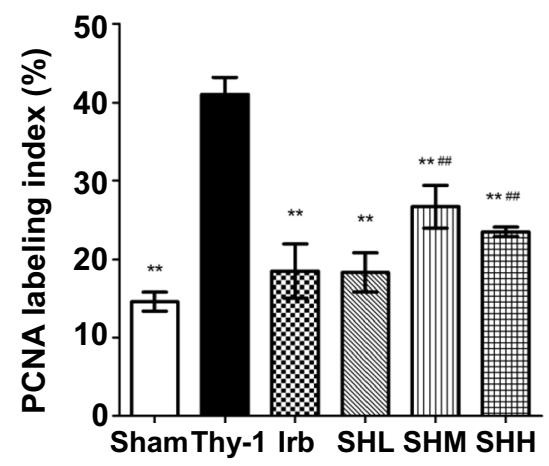

Fig. 4 Shenhua Tablet inhibits proliferation of mesangial cells in rats with anti-Thy-1 nephritis and improves renal pathological scores. a Histological changes in PAS-stained sections at W20 (×400 magnification). Scale bar $50 \mu \mathrm{m}$. b The total number of glomerular cross section in the six groups. c PCNA-positive cells in the groups at W20. Scale bar $50 \mu \mathrm{m}$. d The PCNA labeling index in all six groups. The index was calculated according to the ratio of PCNA positive cells to total glomerular cells. e Histological scores of the renal lesions in all six groups. Values were expressed as mean \pm SD. ${ }^{*} p<0.05,{ }^{* *} p<0.01$ vs. Thy -1

disease (ESRD) [10]. Therefore, inhibition of mesangial cell proliferation is a great strategy to treat proliferative glomerular diseases. Hence, searching for such inhibitors can have important clinical implications.

In the past 20 years, although mesangial cell proliferation signaling pathways have become clearer, specific drugs used to inhibit the proliferation of mesangial cells are still poorly available. Currently, clinical applications are only limited to a few drugs, such as hormones, cytotoxics, and so on. With the development of Chinese medicine theory, a number of classical prescriptions, special prescriptions and drugs, patent medicines, and their extracts or injections have been proved to be effective in the treatment of human diseases in some respects, which indicates traditional Chinese medicine can also be used in the treatment of chronic kidney disease, such as MsPGN. With accumulating evidences and more studies in clinical and basic research, the efficacy of Chinese medicine in the treatment of chronic kidney diseases has been well recognized in the national and international medical field. It has been clear that Chinese medicine and integrative medicine are effective approaches to treat patients with chronic kidney disease (including MsPGN). Data from multi-center, randomized, double-blind and 


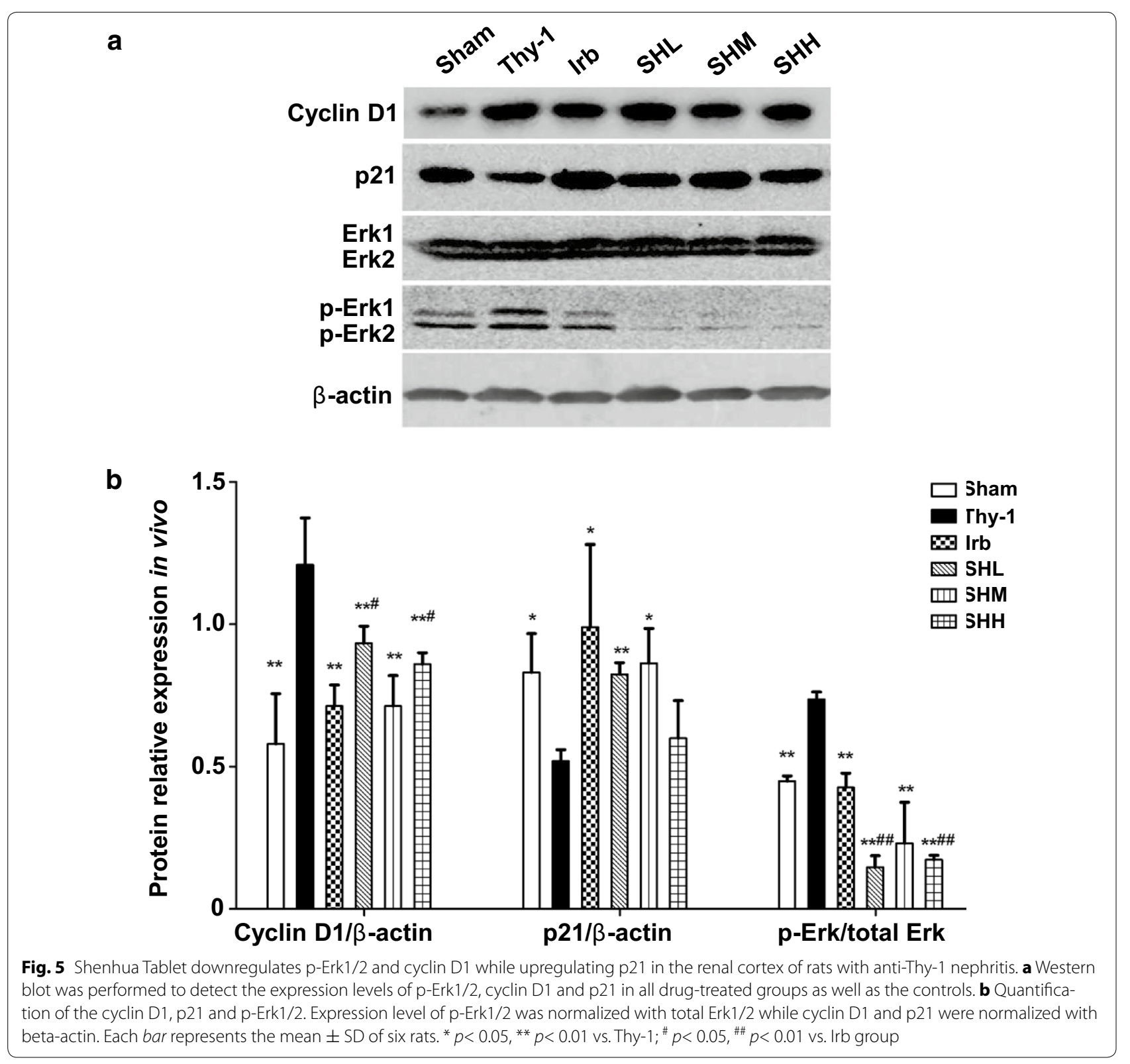

placebo-controlled clinical experiments have shown that Shenhua Tablet is able to reduce proteinuria level in IgA nephropathy patients deficient with Qi and Yin, and to improve their clinical symptoms without serious adverse reactions [7]. However, the mechanism underlying remains poorly understood. To date, only a few studies show that through toll-like receptors (TLRs), treatment of Shenhua Tablet is able to reduce the secretion of inflammatory cytokines, thereby improving the ischemia-reperfusion-induced acute kidney injury [11]. Currently, no experimental data demonstrate whether Shenhua Tablet is able to inhibit mesangial cell proliferation in rats with anti-Thy1 nephritis.
In this study, we first report that Shenhua Tablet is able to inhibit mesangial cell proliferation and protect kidneys in rats with chronic anti-Thy-1 MsPGN, a classic animal model widely used for human MsPGN. The greatest feature of this model is that the renal damages are irreversible, which is different from the acute anti-Thy-1 MsPGN model in which mesangial cell proliferation and matrix accumulation are reversible. To some extent this model can mimic human chronic MsPGN [12], which is an important consideration to improve 'bench to bedside' applicability. However, chronic anti-Thy-1 nephritis in the rat is caused by abnormal deposition of IgG in glomeruli while human IgAN, the most common form of 
MsPGN, is primarily caused by the deposition of IgA in the glomeruli [13], together with the species and genetic differences between rat and human, therefore the results obtained from this model can not be directly used to predict human IgAN. Regardless of this limitation, so far anti-Thy-1 nephritis model is the most representative animal model used to search for drug targets against mesangial cell proliferation [9].

To perform an in vivo study, we orally treated the rats with Shenhua Tablets (low, medium and high dose) once a day for 13 weeks and assessed the drug effect. The data showed that Shenhua Tablets was able to reduce the level of proteinuria and total cholesterol significantly, which was comparable to the therapeutic effect observed in the positive control-irbesartan group. In this study, we noticed that the level of serum creatinine in the rats with anti-Thy-1 nephritis was not significantly reduced when Shenhua Tablet of any dose was applied. In the future study, we may extend the time for drug treatment following the time point of W20, and re-examine if the level of creatinine becomes significant different between the drug-treated group and anti-Thy-1 group. We are also interested in testing whether protein uptake in the proximal tubules is increased in our future studies. We also found that Shenhua Tablet could strikingly inhibit glomerular cell proliferation based on the data obtained from renal pathological scores, glomerular cell counting, PCNA-positive glomerular cell counting, and so on. Previous studies have shown that ERK activity plays an important role in mesangial cell proliferation, and this activity is also essential to cell cycle progression [14, 15]. ERK can translocate into the nucleus, phosphorylate and activate a number of transcription factors, such as AP-1, ELK-1, SAP, and then promote cell proliferation [16]. Once the ERK/MAPK pathway is activated, the downstream target genes, such as cyclin D1, an important regulator of cell cycle at the G1 phase [17], will be activated as well. Furthermore, another important cyclin, termed CDK4, needs to complex with its regulatory subunit cyclin D1 to be functional $[18,19]$. In acute antiThy-1 nephritis model, cyclin D1, CDK4 and other cell cycle-related proteins are all upregulated when mesangial cells are abnormally proliferating. In vitro experiments also confirmed that mesangial cell proliferation was strikingly inhibited when the expression level of cyclin D1 was decreased following removal of the serum or PDGF stimulation [19]. Therefore, we examined one of the key proteins in the Erk/Mapk signaling pathway-Erk1/2, and the cell cycle regulatory proteins in the Shenhua Tablettreated groups. We found that treatment with Shenhua Tablet downregulated the phosphorylation of Erk $1 / 2$ and cyclin D1 while upregulating p21 in the rat model. Therefore, the possible mechanism underlying Shenhua Tablet inhibits the mesangial cell proliferation is that this drug specifically deactivates Erk in the Erk/Mapk pathway, thus downregulates cell cycle regulatory proteins, and ultimately inhibits cell proliferation. These findings have offered a therapeutic potential to Shenhua Tablet in the treatment of chronic glomerulonephritis, particularly for those MsPGN-derived diseases, such as IgAN. In addition, patients with chronic kidney disease often have an abnormal metabolism in blood lipids [20]. Lipids have a direct role in glomerular injury, and may also result in glomerular mesangial cell activation and proliferation [21], and dyslipidemia has been known to be one of the risk factors for the deterioration of renal function. Interestingly, we also found that Shenhua Tablet with different doses could all significantly reduce the levels of both triglycerides and total cholesterol whereas the positive control Irb could only reduce the level of total cholesterol but not of triglycerides. The molecular mechanism underlying this observation needs to be further explored.

\section{Conclusions}

In this study, we discovered that Shenhua Tablet is able to reduce the level of $24 \mathrm{~h}$ proteinuria and the degree of kidney injury in the chronic anti-Thy- 1 nephritis rat model. These functional improvements by Shenhua Tablet treatment in the injured kidneys might be directly associated with the deactivation of Erk signaling pathway, modulations of downstream cell cycle regulatory proteins, thereby inhibiting the abnormal proliferation of mesangial cells.

\section{Methods}

\section{Pharmaceutical preparation}

Compound Shenhua Tablets $(0.6 \mathrm{~g} /$ tablet, extracted from at least $3.095 \mathrm{~g}$ of crude drug) were nicely provided by Hunan Traditional Chinese Medicine Research Institute (Batch No. 20090910). The main components in this tablet include Radix Astragali, Fruit of Glossy Privet, Curcuma and Honeysuckle. The non-standardized product number in People's Liberation Army is F01012 (2006). irbesartan was provided by Hangzhou Sanofi-Aventis Minsheng Pharmaceutical Co., Ltd. (Batch number: 0A008; approval number: Zhunzi J20080061). The drug powder was mixed and suspended with $0.4 \% \mathrm{CMC}-\mathrm{Na}$ (Wako Pure Chemical Industries, Ltd., Japan) solution prior to intragastric administration to rats.

\section{Animal model of chronic anti-Thy- 1 nephritis}

Seven-week-old male Wistar rats (SPF grade, 200 g) were purchased from Beijing Vital River Laboratory Animal Technology Co., Ltd., and were bred in the animal room (cleaning grade) of Experimental Animal Center of the People's Liberation Army General Hospital. All 
animal experiments were conducted in accordance with the provisions of Experimental Animal Committee of the People's Liberation Army General Hospital.

Rats were randomly divided into six groups as followed: (1) Sham control (not nephrectomized, injected with same dose of $1 \times$ PBS); (2) Thy-1 (nephrectomized, injected with Thy-1 antibody); (3) Irb (nephrectomized, injected with $\mathrm{Irb}$ at the dose of $20 \mathrm{mg} / \mathrm{kg}$ body weight); (4) SHL (nephrectomized, injected with Shenhua Tablet at the dose of $0.75 \mathrm{~g} / \mathrm{kg}$ body weight); (5) SHM (nephrectomized, injected with Shenhua Tablet at the dose of $1.5 \mathrm{~g} / \mathrm{kg}$ body weight) and (6) SHH (nephrectomized, injected with Shenhua Tablet at the dose of $3 \mathrm{~g} / \mathrm{kg}$ body weight). Each group has six mice. Seven days after unilateral nephrectomy [22], the mice were given monoclonal Thy-1 antibody $(2.5 \mathrm{mg} / \mathrm{kg})$ via intravenous injection [23, 24], which was performed once a week for 4 weeks [25] to induce chronic MsPGN. Twenty-one days after the last injection of Thy-1 antibody (W7), rats were daily treated with drugs (Irb or Shenhua Tablet) at the dose indicated above by gavage for 13 weeks. At each experimental time point, the Sham group was treated with $\mathrm{CMC}-\mathrm{Na}$ solution alone for 13 weeks as well (Fig. 6).

Biochemical and histopathological approaches were taken to trace the progression of MsPGN for each group after the disease was induced by Thy- 1 antibody. Urinary protein and serum creatinine in $24 \mathrm{~h}$ were tested every 2 weeks. At the 18th week (the 13th week after drug intervention), the $24 \mathrm{~h}$ urine was collected and the rats were then anesthetized with $2 \%$ sodium pentobarbital $(45 \mathrm{mg} /$ $\mathrm{kg}$ ) by intraperitoneal injection. The rats were dissected and $8 \mathrm{ml}$ of blood was collected to assess renal function and biochemical indicators of blood. The kidney was cut into several pieces; One of them was fixed with $4 \%$ paraformaldehyde/PBS for pathological examination and another piece saved for immunostaining was embedded in OCT (Optimal Cutting Temperature compound) and promptly frozen in liquid nitrogen and then stored at $-80{ }^{\circ} \mathrm{C}$; The rest of the kidney was directly frozen in liquid nitrogen and then stored at $-80^{\circ} \mathrm{C}$. The changes in proteinuria, renal function and pathology were examined and the expression levels of p-Erk1/2, cyclin D1 and p21 in the renal cortex were also tested.

\section{Renal pathological examination}

Kidneys were first fixed with $4 \%$ paraformaldehyde/ PBS buffer, dehydrated with ethanol and then embedded in paraffin. The kidneys were sectioned into slices of $4 \mu \mathrm{m}$ thickness and PAS (Periodic Acid-Schiff) staining was performed to examine pathological changes under light microscope. The degree of renal impairment was semi-quantitatively and double-blindly scored by two pathologists following the published scoring system [26-29]. We randomly selected ten separate field for each specimen to evaluate lesions ( $400 \times$ magnification), and averaged the values for the histological score.

\section{Immunohistochemical analysis}

The paraffin sections were fixed on polylysine-coated slides, deparaffined in xylene, and then rehydrated in ethanol of decreasing concentrations, and finally rinsed in water. Endogenous peroxidase was blocked with $3 \%$ hydrogen peroxide at room temperature. The slices were then immersed in sodium citrate buffer $(\mathrm{pH}$ 6.0) and microwaved for $10 \mathrm{~min}$. After being blocked with $1.5 \%$ normal goat serum and incubated at room temperature for $20 \mathrm{~min}$, the sections were stained with PCNA (Abcam; mouse monoclonal; 1: 500 dilution) antibody and incubated overnight at $4{ }^{\circ} \mathrm{C}$. The following day, the unbound primary antibody was washed away with $1 \times$ PBS, then biotin-labeled secondary antibody was added and incubated at room temperature for $40 \mathrm{~min}$. After being washed with $1 \times$ PBS, horseradish peroxidase conjugated streptavidin (Vectastain Elite ABC kit, Vector Labs, USA) was added and incubated at room temperature for an additional $40 \mathrm{~min}$. After PBS wash, the sections were treated with $\mathrm{DAB}$ (chromogenic reagent) for 5 min until the color turned brown and were then restained with hematoxylin as a last step. Kidney sections from three rats at each time point were used to quantify proliferative glomerular cells under high magnification microscope $(400 \times)$ in a blind manner. Ten glomerluli were examined for each section. PCNAlabeling index was calculated as "the number of PCNApositive cells divided by the total number of glomerular cells".

\section{Western blotting}

The kidneys were lysed in RIPA buffer $(50 \mathrm{mmol} / \mathrm{L}$ Tris- $\mathrm{HCl}, 150 \mathrm{mmol} / \mathrm{L} \mathrm{NaCl}, 0.5 \%$ deoxycholate, $1 \%$ Nonidet P-40, $0.1 \%$ SDS, $0.5 \mathrm{mM}$ PMSF, $1 \mu \mathrm{g} / \mathrm{ml}$ leupeptin and $1 \mu \mathrm{g} / \mathrm{ml}$ aprotinin) on ice for $30 \mathrm{~min}$, then centrifuged at $12,000 \mathrm{rpm}$ at $4{ }^{\circ} \mathrm{C}$ for $30 \mathrm{~min}$. The protein concentration was measured with Pierce BCA assay kit (Thermo Fisher Scientific, USA). Sixty to onehundred $\mu \mathrm{g}$ of total protein were loaded and run with $12 \%$ polyacrylamide gel. After electrophoresis, proteins were transferred onto nitrate cellulose membrane. The membrane was first blocked with $5 \%$ skim milk at room temperature for $1 \mathrm{~h}$, and then incubated overnight at $4{ }^{\circ} \mathrm{C}$ with the primary antibodies against p-Erk1/2 (mouse monoclonal, 1: 1000 dilution; Beyotime Institute of Biotechnology, China), cyclin D1 (mouse monoclonal, 1: 100 dilution; Santa Cruz, USA), p21 (mouse 
Time after disease induction (week)

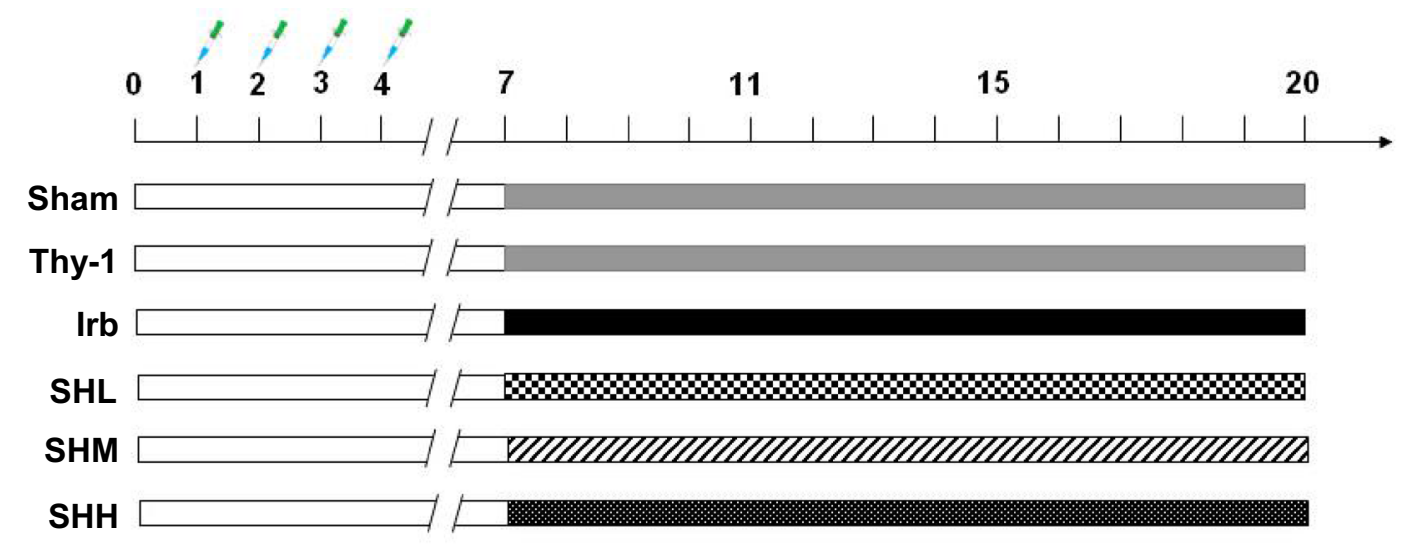

\section{Induction}

W0: uninephrectomized

W1, 2, 3, 4: anti-Thy-1 antibody (2.5mg/kg body weight) W7-W20: Treatment

\begin{tabular}{|c|c|c|}
\hline & Placebo & \\
\hline & & $20 \mathrm{mg} / \mathrm{kg}$ b \\
\hline & SHL & $0.75 \mathrm{~g}$ \\
\hline & SHH & $\begin{array}{l}1.00 \\
3.00\end{array}$ \\
\hline
\end{tabular}

Fig. 6 A scheme of drug treatment for the animal model of chronic anti-Thy-1 MsPGN. Thirty-six Wistar rats were randomly divided into six groups: (1) Sham surgery group (Sham); (2) anti-Thy-1 nephritis model group (Thy-1); (3) anti-Thy-1 nephritis model + irbesartan-treated group (Irb); (4) antiThy-1 nephritis model + low-dose of Shenhua Tablet group (SHL); (5) anti-Thy-1 nephritis model + medium-dose of Shenhua Tablet group (SHM); (6) anti-Thy-1 nephritis model + high-dose of Shenhua Tablet group (SHH)

monoclonal, 1: 5000 dilution; Novus Biologicals, USA) or $\beta$-actin (mouse monoclonal, 1: 5000 dilution; Sigma, USA) respectively. After incubation of individual primary antibody, the membrane was washed three times with TBST (Tris-Buffered Saline plus Tween 20) followed by the incubation of secondary antibody for $1 \mathrm{~h}$. The membrane was washed with TBST again and was then detected using an enhanced chemiluminescence (ECL) method. The intensity of proteins was quantified with Quantity One software (Bio-Rad, USA). All experiments were repeated at least three times.

\section{Statistical analysis}

All data were processed with SPSS 15.0 software (SPSS Inc. USA). Charts were generated with Excel 2003. Data from continuous variables were expressed with $(\bar{x} \pm \mathrm{s})$. The comparison between numerical variables was performed with $t$ test or ANOVA; Values were expressed as mean \pm SD (Standard Deviation); $p<0.05$ means statistically significant.

\section{Abbreviations}

MsPGN: mesangial proliferative glomerulonephritis; Irb: irbesartan; ESRD: end-stage renal disease; PCNA: proliferating cell nuclear antigen; TLRs: toll-like receptors; SHL: low-dose of Shenhua Tablet; SHM: medium-dose of Shenhua Tablet; SHH: high-dose of Shenhua Tablet.

\section{Authors' contributions}

WJG and XMC conceived and designed the experiments. WJG, RBW, SWL, LT, $H Y Z, P C, J W, X G Z, F Z$, and $Z Y$ performed the experiments and analyzed the data. WJG, RBW and XMC wrote the paper. All authors read and approved the final manuscript.

\section{Author details}

${ }^{1}$ Department of Nephrology, Chinese PLA General Hospital, Chinese PLA Institute of Nephrology, State Key Laboratory of Kidney Diseases, National Clinical Research Center for Kidney Diseases, Beijing Key Laboratory of Kidney Disease Research, Beijing 100853, People's Republic of China. ${ }^{2}$ Department of Nephrology, Guangdong Provincial Hospital of Chinese Medicine, Guangzhou 510120, People's Republic of China.

\section{Acknowledgements}

This work was supported by the Programs of the National Natural Science Foundation of China (Grant No. 81330019), China Postdoctoral Science Foundation (Grant No. 2013M542447) and the National Natural Science Foundation of China (Grant No. 61471399). The funders are not involved in the experimental design, data collection and analysis, and manuscript preparation. 


\section{Competing interests}

All authors declare that they have no competing interests.

Received: 8 October 2015 Accepted: 2 March 2016 Published online: 11 March 2016

\section{References}

1. Zhou FD, Zhao MH, Zou WZ, Liu G, Wang H. The changing spectrum of primary glomerular diseases within 15 years: a survey of 3331 patients in a single Chinese centre. Nephrol Dial Transplant. 2009;24:870-6.

2. Nair R, Walker PD. Is IgA nephropathy the commonest primary glomerulopathy among young adults in the USA? Kidney Int. 2006;69:1455-8.

3. Couser WG, Johnson RJ. Mechanisms of progressive renal disease in glomerulonephritis. Am J Kidney Dis. 1994;23:193-8.

4. Kashgarian M, Sterzel RB. The pathobiology of the mesangium. Kidney Int. 1992;41:524-9.

5. Xie Y, Chen X. Epidemiology, major outcomes, risk factors, prevention and management of chronic kidney disease in China. Am J Nephrol. 2008;28:1-7.

6. Kawasaki Y. The pathogenesis and treatment of IgA nephropathy. Fukushima J Med Sci. 2008;54:43-60.

7. Chen X, Chen Y, Chen J. Multicentered, randomized, controlled clinical trial on patients with IgA nephropathy of Qi-yin deficiency syndrome type. Chin J Integr Med. 2007;27:101-5.

8. Bagchus WM, Jeunink MF, Elema JD. The mesangium in anti-Thy-1 nephritis. Influx of macrophages, mesangial cell hypercellularity, and macromolecular accumulation. Am J Pathol. 1990;137:215-23.

9. Jefferson JA, Johnson RJ. Experimental mesangial proliferative glomerulonephritis (the anti-Thy-1.1 model). J Nephrol. 1999;12:297-307.

10. Xie J, Chen N. Primary glomerulonephritis in mainland China: an overview. Contrib Nephrol. 2013;181:1-11.

11. Zheng XY, Wei RB, Shi SZ, Yin Z, Chen XM. Effects of Fufang Shenhua Tablet on the expression of toll-like receptors during acute kidney injury induced by ischemia-reperfusion in rats. Chin J Integr Med. 2012;18:918-24.

12. Morita H, Maeda K, Obayashi M, Shinzato T, Nakayama A, Fujita Y, et al. Induction of irreversible glomerulosclerosis in the rat by repeated injections of a monoclonal anti-Thy-1.1 antibody. Nephron. 1992;60:92-9.

13. Li LS, Liu ZH. Epidemiologic data of renal diseases from a single unit in China: analysis based on 13,519 renal biopsies. Kidney Int. 2004;66:920-3.

14. Pages G, Lenormand P, L'Allemain G, Chambard JC, Meloche S, Pouyssegur J. Mitogen-activated protein kinases p42mapk and p44mapk are required for fibroblast proliferation. Proc Natl Acad Sci USA. 1993;90:8319-23.
15. Schramek H, Schumacher M, Pfaller W. Sustained ERK-2 activation in rat glomerular mesangial cells: differential regulation by protein phosphatases. Am J Physiol. 1996;271:F423-32.

16. Boilly B, Vercoutter-Edouart AS, Hondermarck H, Nurcombe V, Le Bourhis $X$. FGF signals for cell proliferation and migration through different pathways. Cytokine Growth Factor Rev. 2000;11:295-302.

17. Doan CC, Le LT, Hoang SN, Do SM, Le DV. Simultaneous silencing of VEGF and KSP by siRNA cocktail inhibits proliferation and induces apoptosis of hepatocellular carcinoma Hep3B cells. Biol Res. 2014;47:70.

18. Nakano K, Chijiiwa K, Tanaka M. Lower activity of CCAAT/enhancerbinding protein and expression of cyclin E, but not cyclin D1, activating protein-1 and p21(WAF1), after partial hepatectomy in obstructive jaundice. Biochem Biophys Res Commun. 2001;280:640-5.

19. Schocklmann HO, Lang S, Sterzel RB. Regulation of mesangial cell proliferation. Kidney Int. 1999;56:1199-207.

20. Fellstrom B, Holdaas H, Jardine AG, Svensson MK, Gottlow M, Schmieder $\mathrm{RE}$, et al. Cardiovascular disease in patients with renal disease: the role of statins. Curr Med Res Opin. 2009;25:271-85.

21. Ezquer ME, Ezquer FE, Arango-Rodriguez ML, Conget PA. MSC transplantation: a promising therapeutic strategy to manage the onset and progression of diabetic nephropathy. Biol Res. 2012;45:289-96.

22. Tsuji M, Monkawa T, Yoshino J, Asai M, Fukuda S, Kawachi H, et al. Microarray analysis of a reversible model and an irreversible model of anti-Thy-1 nephritis. Kidney Int. 2006;69:996-1004.

23. Lu Y, Liu X, Shi S, Su H, Bai X, Cai G, et al. Bioinformatics analysis of proteomic profiles during the process of anti-Thy 1 nephritis. Mol Cell Proteomics. 2012;11(M111):008755.

24. Liu X, LV Y, Xie Y, Hong Q, Cai G, Zhang S, et al. Change of MAX interactor 1 expression in an anti-Thy 1 nephritis model and its effect on mesangial cell proliferation. Cell Physiol Biochem. 2011;27:391-400.

25. Harendza S, Schneider A, Helmchen U, Stahl RA. Extracellular matrix deposition and cell proliferation in a model of chronic glomerulonephritis in the rat. Nephrol Dial Transplant. 1999;14:2873-9.

26. Jiang L, Liu G, LV J, Huang C, Chen B, Wang S, et al. Concise semiquantitative histological scoring system for immunoglobulin A nephropathy. Nephrology (Carlton). 2009;14:597-605.

27. Katafuchi R, Kiyoshi Y, Oh Y, Uesugi N, Ikeda K, Yanase T, et al. Glomerular score as a prognosticator in IgA nephropathy: its usefulness and limitation. Clin Nephrol. 1998;49:1-8.

28. Shigematsu H. Histological grading and staging of IgA nephropathy. Pathol Int. 1997;47:194-202.

29. Austin HA 3rd, Muenz LR, Joyce KM, Antonovych TT, Balow JE. Diffuse proliferative lupus nephritis: identification of specific pathologic features affecting renal outcome. Kidney Int. 1984;25:689-95.

\section{Submit your next manuscript to BioMed Central and we will help you at every step:}

- We accept pre-submission inquiries

- Our selector tool helps you to find the most relevant journal

- We provide round the clock customer support

- Convenient online submission

- Thorough peer review

- Inclusion in PubMed and all major indexing services

- Maximum visibility for your research

Submit your manuscript at www.biomedcentral.com/submit

\section{Biomed Central}

